\title{
Defects Responsible for Hydrogen Embrittlement in Austenitic Stainless Steel 304 by Positron Annihilation Lifetime Spectroscopy
}

\author{
Luca CHIARI, ${ }^{*}$ Akari KOMATSU and Masanori FUJINAMI \\ Department of Applied Chemistry and Biotechnology, Chiba University, 1-33 Yayoi, Inage, Chiba, 263-8522 Japan.
}

(Received on November 2, 2020; accepted on January 26, 2021)

\begin{abstract}
Hydrogen-related defects in the metastable austenitic stainless steel 304 were analyzed by positron annihilation lifetime spectroscopy to determine the factors responsible for hydrogen embrittlement. Hydrogen was introduced by the cathodic electrolysis method to the $\sim 10-\mu \mathrm{m}$ topmost layer which was then etched by electrochemical polishing to investigate the formation of hydrogen-induced defects in the bulk. Although hydrogen embrittlement did not occur simply upon removal of the hydrogen-charged layer, a decrease in ductility was confirmed by applying $10 \%$ tensile strain and subsequent polishing. In this latter sample a positron lifetime component of $\sim 180$ ps was detected, which is longer than that of dislocations, and disappeared upon annealing at $100^{\circ} \mathrm{C}$. After further straining the sample until fracture, the formation of vacancy clusters was observed. These results suggest that hydrogen diffused to deeper regions upon application of tensile stress, where vacancy-hydrogen complexes were generated and developed into vacancy agglomerates. The detection of the precursors of the vacancy clusters, which are thought to lead to the brittle fracture, represents a fundamental step forward in the understanding of the hydrogen embrittlement process in austenitic stainless steels.
\end{abstract}

KEY WORDS: hydrogen embrittlement; austenitic stainless steel; positron annihilation lifetime spectroscopy; vacancy defect.

\section{Introduction}

In recent years, in response to global issues such as environmental sustainability and fossil fuel depletion, the use of hydrogen as an alternative energy source has become increasingly widespread. ${ }^{1)}$ At the same time, high strength and light weight steels are widely required by industry in the effort to reduce carbon dioxide emissions and for resource conservation. ${ }^{2)}$ Hydrogen embrittlement (HE) constitutes a critical issue in the implementation of these large-scale projects. HE is a phenomenon that causes the deterioration of the mechanical properties, e.g. a decrease in ductility and early fracture, of susceptible metals such as iron and steels when exposed to hydrogen. The HE mechanism has been debated for many years but despite extensive applied research and phenomenological theories, a unified view of the lattice defects and the controlling factors has not yet been reached. ${ }^{3)}$ If this process is elucidated, it will be possible to overcome the HE of mechanical and structural materials, and provide design guidelines for new alloys and microstructure control that contribute to improved safety

\footnotetext{
* Corresponding author: E-mail: luca.chiari@chiba-u.jp
}

and cost reduction. ${ }^{4)}$

Dislocations, stacking faults, and atomic vacancies have been proposed as the potential controlling factors of HE. ${ }^{3)}$ Positron annihilation lifetime spectroscopy (PALS) positions itself as the ideal non-destructive tool for measurements of the size and concentration of such atomic defects even on the ppm order. ${ }^{5)}$ In $\alpha$-iron, hydrogen promotes the vacancy formation by stabilizing the monovacancies generated by the interaction between dislocations during the plastic deformation. ${ }^{6)}$ PALS measurements have demonstrated that these hydrogen-induced vacancies agglomerate into vacancy clusters. ${ }^{7,8)}$ The formation of vacancy clusters requires the presence of their precursor, the vacancy-hydrogen complex (i.e. a monovacancy stabilized by trapped hydrogen atoms). ${ }^{9}$

Austenitic stainless steels are often used as structural materials in the hydrogen energy infrastructure. So far, a significant amount of phenomenological data has been collected, but the fundamental mechanism of HE remains unclear. ${ }^{10)}$ It is acknowledged that HE is remarkable in metastable austenitic stainless steels, ${ }^{11)}$ such as the 304 grade, whereas it is greatly reduced in austenitic stainless steels with a high Ni-equivalent, e.g. in the stable austenitic stainless steel 316 L. ${ }^{12,13)}$ XRD measurements have shown 
that hydrogen adsorption in austenitic stainless steels with low $\gamma$-phase stability (e.g. 304) causes a phase transformation from the austenite phase to the $\varepsilon$ and $\alpha^{\prime}$ martensite phases and the formation of austenite hydrides. ${ }^{14,15)}$ This demonstrates that HE is greatly dependent on the stability of the $\gamma$-phase, namely increasing the $\gamma$-phase stability reduces HE in austenitic stainless steels. ${ }^{12)}$ Nonetheless, HE was found to occur in 304 steel even when no strain-induced $\alpha^{\prime}$-phase was observed. ${ }^{16)}$ Hence, the formation of the straininduced $\alpha^{\prime}$-phase is not the direct cause of HE.

In austenitic stainless steels, the relationship between microstructure, lattice defects and hydrogen is a fundamental factor to understand the HE process. ${ }^{17}$ ) There is ample evidence that hydrogen enhances the velocity of dislocations. ${ }^{18)}$ PALS measurements have shown that the interaction between dislocations and hydrogen enhances the formation of strain-induced vacancies which aggregate into vacancy clusters in 304 and 316 L stainless steels. ${ }^{16,19)}$ These vacancy clusters are believed to accumulate locally in high-strain regions, such as the boundaries between the $\gamma$-phase and the $\alpha^{\prime}$-phase, and become the starting point of the brittle fracture. ${ }^{20,21)}$ However, vacancy clusters were found to form in a hydrogen environment regardless of whether HE occurs or not, ${ }^{16)}$ so that their mere presence cannot be regarded the critical factor of HE. Although the role of strain-induced vacancies is understood to be important in the HE of austenitic stainless steels, ${ }^{22}$ many questions about the enhanced formation of vacancies, as a result of the interaction between hydrogen and lattice defects, remain unanswered. The main reason for this lies in the hydrogen diffusion coefficient in the austenitic $\gamma$-phase, which is 6 order of magnitudes smaller than in $\alpha$-iron. Due to the slow diffusion of hydrogen in the $\gamma$-phase, defect analysis in austenitic stainless steels by thermal desorption spectroscopy remains quite limited, ${ }^{23,24)}$ and other analytical techniques such as PALS are warranted.

The HE susceptibility in austenitic stainless steels is known to increase in severe environments such as under high-pressure hydrogen gas or at low temperatures. ${ }^{3)}$ The temperature dependence of HE suggests that the hydrogen diffusion and fugacity are as important as the stability of the $\gamma$-phase. HE also occurs in austenitic stainless steel 304 when hydrogen is added by cathodic electrolysis. ${ }^{25)}$ In this case, hydrogen is introduced only in the surface layer and yet the ductility reduction is induced throughout the bulk. It is anticipated that hydrogen diffuses to deeper regions inside the sample by applying stress, and that the defect formation is dependent on the depth, but no empirical evidence to support these assumptions exists to date.

In this paper, a detailed analysis of the hydrogen-related defects in austenitic stainless steel 304 was conducted by taking advantage of the high defect sensitivity of PALS. In austenitic stainless steels, the $\varepsilon$ and $\alpha^{\prime}$-phases are formed in the topmost layer upon hydrogen addition ${ }^{14,15)}$ and the properties of this layer are significantly different from those of the bulk. To distinguish the defects formed in the upmost layer from those in the bulk, the phase structure formed in the $10-\mu \mathrm{m}$ topmost layer was removed by electropolishing and the samples were characterized by tensile testing, XRD, SEM and PALS measurements.

\section{Experimental Details}

\subsection{Sample Preparation}

An austenitic stainless steel 304 thin plate was used as the source for the specimens. The chemical composition is specified in Table 1 and the average grain size is $1 \mu \mathrm{m}$. Dumbbell-shaped samples (Fig. 1) were processed by electric discharge machining. The width of the samples is 10 $\mathrm{mm}$, the total and gauge lengths are $45 \mathrm{~mm}$ and $20 \mathrm{~mm}$, respectively, and the thickness is $0.2 \mathrm{~mm}$. A notch (semicircle shape, radius $1 \mathrm{~mm}$ ) was placed at the center of the samples to concentrate the tensile stress in the fracture position in order to obtain two identical copies of each specimen for the PALS measurements (see below).

The samples underwent solution treatment in Ar atmosphere using an infrared gold image furnace to remove the initial defects introduced during rolling and straining of the material. The heating rate was $4^{\circ} \mathrm{C} / \mathrm{min}$ over a period of 4 hours until the annealing temperature of $900^{\circ} \mathrm{C}$ was reached. After annealing at that temperature for $5 \mathrm{~min}$, the temperature was gradually reduced to room temperature (RT). The crystal structure of stainless steel 304 after annealing was confirmed by XRD measurements and in previous studies ${ }^{16)}$ employing the same heat treatment. During the solution treatment, a several $\mu \mathrm{m}$-thick black oxide layer was formed on the surface of the samples and was removed by mechanical and chemical polishing. Preliminary PALS measurements after removal of the oxide layer showed the presence of a single lifetime component with a value consistent with that of steel bulk ( $\sim 110 \mathrm{ps})$, confirming the absence of defects in the samples.

\subsection{Hydrogen Charge}

Hydrogen was introduced in the samples by the electrolytic cathodic charging method. ${ }^{25}$ ) The electrolyte was an aqueous solution of 3 mass $\% \mathrm{NaCl}+3 \mathrm{~g} / 1 \mathrm{NH}_{4} \mathrm{SCN}$ and the charge was conducted at a constant electric current density of $50 \mathrm{~A} / \mathrm{m}^{2}$ for 48 hours at $\mathrm{RT}$. The section of the sample where hydrogen was introduced has a gauge length of $20 \mathrm{~mm}$ and a width of $10 \mathrm{~mm}$. Inspection of the

Table 1. Chemical composition (mass\%) of austenitic stainless steel 304 .

\begin{tabular}{ccccccccc}
\hline $\mathrm{C}$ & $\mathrm{Si}$ & $\mathrm{P}$ & $\mathrm{S}$ & $\mathrm{Cr}$ & $\mathrm{Mn}$ & $\mathrm{Ni}$ & $\mathrm{Mo}$ & $\mathrm{Fe}$ \\
\hline 0.059 & 0.56 & $<0.045$ & $<0.03$ & 18.8 & $<2$ & 8.2 & 0.2 & balance \\
\hline
\end{tabular}

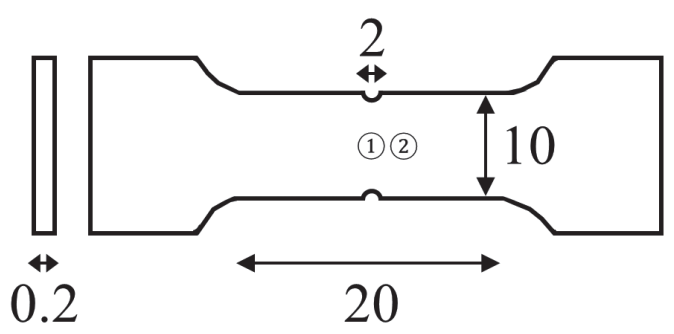

Fig. 1. Schematic diagram of the specimen shape and size. (1) and (2) indicate the locations of the positron source for the PALS measurements on the unstrained and 10\%-prestrained samples, and on the fractured samples, respectively. Units: $\mathrm{mm}$. 
surface condition of the specimens after charge confirmed the absence of surface cracks or blistering as a result of the charge itself. Under these charging conditions, the concentration of adsorbed hydrogen in the sample tends to decrease exponentially with the depth from the surface due to the short hydrogen diffusion length in $\gamma$-iron, and hydrogen is mostly concentrated in the $10-\mu \mathrm{m}$ topmost layer. ${ }^{26)}$ Based on the measured hydrogen diffusion coefficient in austenitic stainless steel 304, the adsorbed hydrogen concentration and the exposure time, ${ }^{13)}$ the hydrogen concentration in the uppermost layer was calculated to be about 172 ppm, whereas in the bulk was just $\sim 6 \mathrm{ppm}$. These values were confirmed through preliminary measurements of the hydrogen content before and after removal of the hydrogencharged layer by thermal desorption analysis. In metastable austenitic stainless steels, it was reported that HE occurs at hydrogen concentrations as low as $\sim 10$ ppm. ${ }^{27)}$ Therefore, the $10-\mu \mathrm{m}$ charged layer is enough to induce the embrittlement of the bulk.

\subsection{Tensile Testing}

After hydrogen charging, the samples were subject to tensile testing using a tensile tester (DEBEN Microtest 2 $\mathrm{kN}$ ) with a maximum load of $2000 \mathrm{~N}$ and a maximum displacement of $20 \mathrm{~mm}$. The tensile strain rate was $4.17 \times$ $10^{-4} / \mathrm{s}$ (gauge length: $20 \mathrm{~mm}$, stroke speed: $0.5 \mathrm{~mm} / \mathrm{min}$ ) for all samples. This strain rate value was chosen after tensile tests with strain rates ranging from $4.17 \times 10^{-5} / \mathrm{s}$ to $4.17 \times$ $10^{-2} / \mathrm{s}$. Like pure iron, austenitic stainless steels might also exhibit a HE susceptibility dependence on the strain rate. ${ }^{28,29)}$ Nonetheless, from those preliminary measurements it was understood that the HE of the 304 steel used in this study had a relatively small strain rate dependence and, hence, it was neglected. Tensile testing was carried out until fracture except for the pre-strained samples, which were first strained up to $10 \%$, released once, and then again deformed till fracture.

\subsection{Electrochemical Polishing}

To remove the topmost layer consisting of the straininduced transformed martensite phase due to the hydrogen addition, chemical etching of the surface was carried out by electrolytic polishing ${ }^{30)}$ to avoid the generation of strain-induced defects. The electropolishing solution was a $\mathrm{H}_{2} \mathrm{SO}_{4}: \mathrm{H}_{3} \mathrm{PO}_{4}=2: 3$ (volume ratio) mixed solution and the electrolytic polishing was carried out at RT with at a current of $4 \mathrm{~A}$. To polish only the gauge section of the samples where hydrogen was introduced, the grip sections were covered with nail polish. The thickness reduction of the specimens due to the chemical etching was confirmed with a micrometer at three points, namely at the center (notch) and at $5 \mathrm{~mm}$ to the left and right from the center. The electropolishing was continued until a layer of $\sim 10 \mu \mathrm{m}$ thickness was removed. Care was taken to ensure that the sample surface was uniformly polished to within $\sim 1 \mu \mathrm{m}$ uncertainty.

\subsection{Positron Annihilation Lifetime Measurements}

PALS is a non-destructive very-sensitive technique for the detection of vacancy-type defects in a large variety of solid materials. ${ }^{5)}$ PALS measurements were carried out with a digital PALS spectrometer composed of two photo- multiplier tubes coupled to $\mathrm{BaF}_{2}$ scintillators connected to a digital storage oscilloscope (Teledyne LeCroy WaveRunner $606 \mathrm{Zi}$ ). The resolution function of the spectrometer is $\sim 180 \mathrm{ps}$ (full width at half maximum). A $0.2 \mathrm{MBq}{ }^{22} \mathrm{Na}$ radioisotope embedded between two $7.5-\mu \mathrm{m}$ thick Kapton foils was used as the positron source. The size of the source was estimated to be $\sim 3 \mathrm{~mm}$ in diameter. Measurements on the unstrained and $10 \%$ pre-strained specimens were performed by sandwiching the source in between two identical copies of the same specimen and placing the source in the middle of the gauge section (position (1) as indicated in Fig. 1). For measurements on the fractured samples, the source was sandwiched in between each half of the specimen and positioned a few mm away from the fracture (position (2) as indicated in Fig. 1) so that the positrons probed the gauge section near the fracture. Positron lifetime spectra were collected by accumulating more than 1 million positron annihilation events in each spectrum to achieve enough statistical accuracy. Each spectrum was measured at least three times to ensure the reproducibility of the data and each measurement was repeated multiple times to confirm repeatability. This approach was successfully employed in previous PALS studies by our and other groups. ${ }^{7,8,16,21)}$

Analysis of the measured spectra was performed by least-squares fitting with the software PALSfit. ${ }^{31)}$ Before the fitting, the background and source component stemming from positron annihilation in the Kapton film were subtracted from each spectrum. The source component (380 ps, 14\%) was well characterized through measurements on reference materials such as annealed pure iron and stainless steel 304. The spectra were fitted with the sum of a number of exponential decay functions so that the $\chi^{2}$ values were smaller than 1.2 to ensure the accuracy of the fits. The positron lifetime of each component reflects the type and size of defects, while the intensity provides their relative concentration. From first principle calculations of the positron lifetime in iron, ${ }^{32,33)}$ the lifetime value for typical defect species is known: $\sim 110$ ps in the bulk, $\sim 160$ ps at dislocations, $\sim 180 \mathrm{ps}$ in monovacancies and $>200$ ps in vacancy clusters. Those computations also yield the relationship between the positron lifetime and the size of vacancy clusters, namely the number of atomic vacancies in the cluster, which allows one to infer the average size of vacancies from the measured lifetime. By applying strain, a lifetime in the range $\sim 140-175 \mathrm{ps}$ is often detected due to the combination of dislocations with monovacancies by the dislocation motion and interaction. Hereafter, defects with such a lifetime will simply be labeled "dislocations".

Total uncertainties on the measured positron lifetimes and the respective intensities account for the statistical uncertainties of the spectrum deconvolution and resolution function of each measurement. For further details on the PALS technique the reader is refereed to reference. ${ }^{5)}$ Also note that the mean positron penetration depth in iron-based alloys is $\sim 100 \mu \mathrm{m}$, whereas the hydrogen diffusion length is less than $10 \mu \mathrm{m}$. Since there is a hydrogen distribution in the samples, a non-uniform defect distribution is expected. The present PALS measurements include information on both regions that contain a large amount of hydrogen and regions that do not. Consequently, these results could generally not be interpreted using the trapping model. ${ }^{34)}$ Nonetheless, 
there are several earlier investigations in the literature ${ }^{7,8,16,21)}$ which proved the effectiveness of the radioisotope-based PALS technique at examining hydrogen-related defects in various hydrogen-susceptible metals.

\section{Results and Discussion}

Figure 2 shows the stress-strain curves of the hydrogenfree, hydrogen-charged, and hydrogen-charged and $10-\mu \mathrm{m}$ etched samples. The HE of the hydrogen-charged specimen is manifest from the significant reduction in ductility compared to the hydrogen-free sample. After hydrogen charge and $10-\mu \mathrm{m}$ etching, the ductility does not decrease. This means that HE does not occur if just $\sim 6 \mathrm{ppm}$ of solute hydrogen is present in the material, in contrast with earlier results. $^{27)}$

The SEM images of the fracture surface of the hydrogenfree and hydrogen-charged samples are shown in Figs. 3(a) and 3(b). Dimples characteristic of a ductile fracture are visible inside both samples. However, near the surface side of the hydrogen-charged sample a random fracture pattern different from dimples was formed, and resembles a quasi-cleavage characteristic of HE. Such fracture pattern is observed up to a depth of 15-20 $\mu \mathrm{m}$ from the surface, which almost coincides with the hydrogen-enriched layer.

The crystal structure and phase of these samples was characterized by XRD measurements (Fig. 4). In the hydrogenfree fractured sample, the presence of the $\alpha^{\prime}\left(\begin{array}{lll}1 & 1 & 0\end{array}\right)$ peak

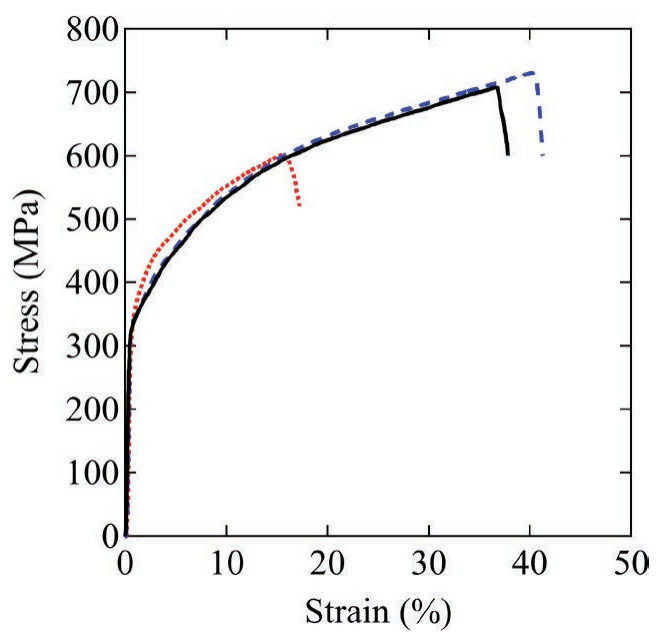

Fig. 2. Stress-strain curves of the ( $\longrightarrow$ ) hydrogen-free, (.........) hydrogen-charged, and (- - -) hydrogen-charged and $10-\mu \mathrm{m}$ etched samples. (Online version in color.) unlike in the undeformed reference sample indicates the formation of the strain-induced $\alpha^{\prime}$-martensite phase. In addition, the broadening of the $\gamma\left(\begin{array}{lll}1 & 1 & 1\end{array}\right)$ peak reflects the lattice distortion and the abundant creation of dislocations upon straining. In the hydrogen-charged sample, both $\gamma\left(\begin{array}{lll}1 & 1 & 1\end{array}\right)$ and $\gamma\left(\begin{array}{lll}2 & 0 & 0\end{array}\right)$ peaks are shifted to lower diffraction angles and become larger in width due to the expansion of the lattice by the introduction of hydrogen. More importantly, the appearance of weak $\alpha^{\prime}\left(\begin{array}{lll}1 & 1 & 0\end{array}\right)$ and $\varepsilon\left(\begin{array}{lll}1 & 0 & 1\end{array}\right)$ peaks demonstrates the formation of hydrides, namely $\alpha^{\prime}$ and $\varepsilon$ martensite phases, due to the high concentration of hydrogen in the upmost layer. The reason why the martensite phase is formed even in the unstrained state is thought to be the stress generated by the lattice expansion due to solid solution hydrogen. These peaks become stronger in intensity after the hydrogen-charged sample is fractured, which indicates that the strain application promotes the formation of the $\alpha^{\prime}$ and $\varepsilon$-phases. After etching the $10-\mu \mathrm{m}$ topmost layer of the hydrogen-charged fractured specimen, the disappearance of the $\alpha^{\prime}\left(\begin{array}{lll}1 & 1 & 0\end{array}\right)$ and $\varepsilon\left(\begin{array}{lll}1 & 0 & 1\end{array}\right)$ peaks indicates that the $\alpha^{\prime}$ and $\varepsilon$-phases are formed only in the hydrogen-charged layer. Hence, it can be concluded that most of these phases are hydrogen induced and that the strain-induced martensite phase is not expected to form up to $\sim 16 \%$ strain. Although not shown in Fig. 4, the hydrogen-charged, $10-\mu \mathrm{m}$ etched, fractured specimen shows a similar pattern to the hydrogenfree fractured sample.

The results of the PALS measurements on these three fractured samples are shown in Fig. 5. For comparison, the hydrogen-charged sample was $10-\mu \mathrm{m}$ etched after fracture and those PALS results are also reported. Although not shown in Fig. 5, we note here that the hydrogen charge itself does not alter the lifetime from the bulk value, which indicates that the hydride formation has little effect on the positron lifetime. In the hydrogen-free fractured sample a single positron lifetime component of $\sim 160$ ps is observed, corresponding to the formation of a large number of straininduced dislocations. The deformation till fracture generates a high concentration of defects which trap all positrons (full trap) and, therefore, no bulk component is observed. On the other hand, in the hydrogen-charged fractured material, the presence of a second component with a lifetime of 335 ps was also detected. This long lifetime value is ascribed to the formation of vacancy clusters with a size of around 13 atomic vacancies based on first-principle calculations. ${ }^{33)}$ Since in the absence of hydrogen vacancy clusters are normally not detected upon application of tensile strain even after fracture, this result confirms the generation of
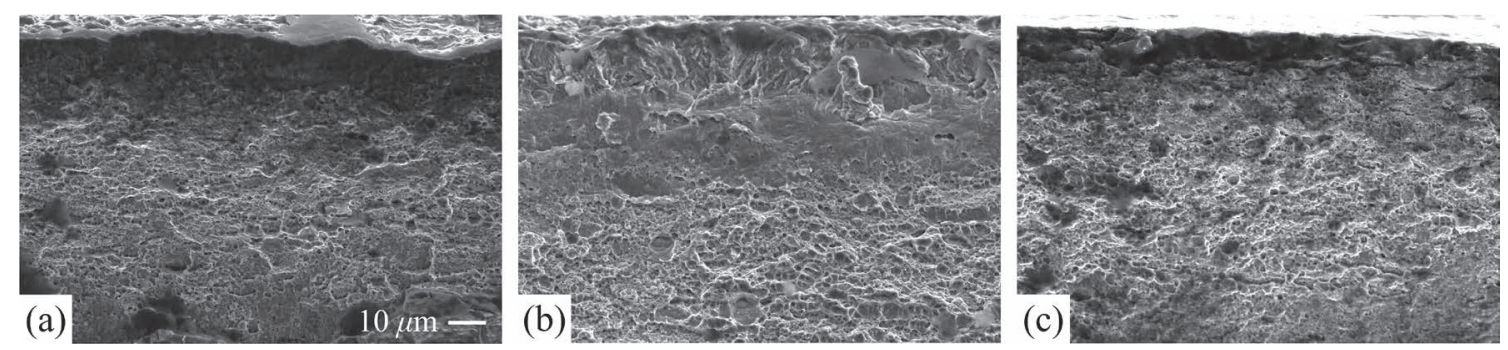

Fig. 3. SEM images of the fracture surfaces of the (a) hydrogen-free, (b) hydrogen-charged, and (c) hydrogen-charged $10 \%$ pre-strained and $10-\mu \mathrm{m}$ etched samples. The upper part of the images shows the sample surface side. The magnification is $1000 \times$. 


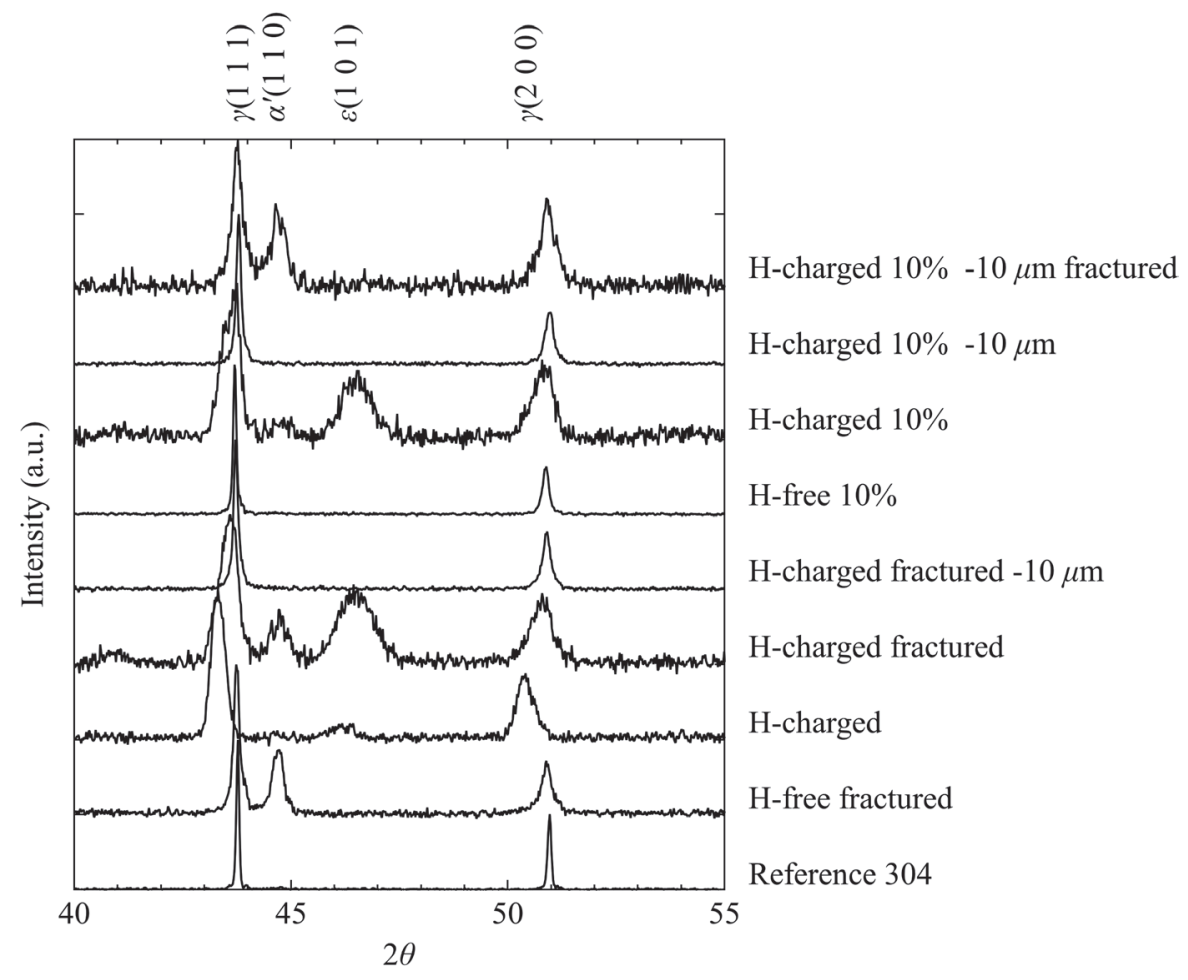

Fig. 4. XRD spectra normalized to the intensity of the $\gamma\left(\begin{array}{lll}1 & 1 & 1\end{array}\right)$ peak. The phase attributed to each peak is shown above the graph. From bottom to top: reference (annealed) austenitic stainless steel 304; hydrogen-free fractured sample; hydrogen-charged sample (undeformed); hydrogen-charged and fractured sample; hydrogen-charged, fractured and $10-\mu \mathrm{m}$ etched sample; hydrogen-free and $10 \%$ pre-strained sample; hydrogen-charged and $10 \%$ pre-strained sample; hydrogen-charged, $10 \%$ pre-strained and $10-\mu \mathrm{m}$ etched sample; hydrogen-charged, $10 \%$ prestrained, $10-\mu \mathrm{m}$ etched and fractured sample.
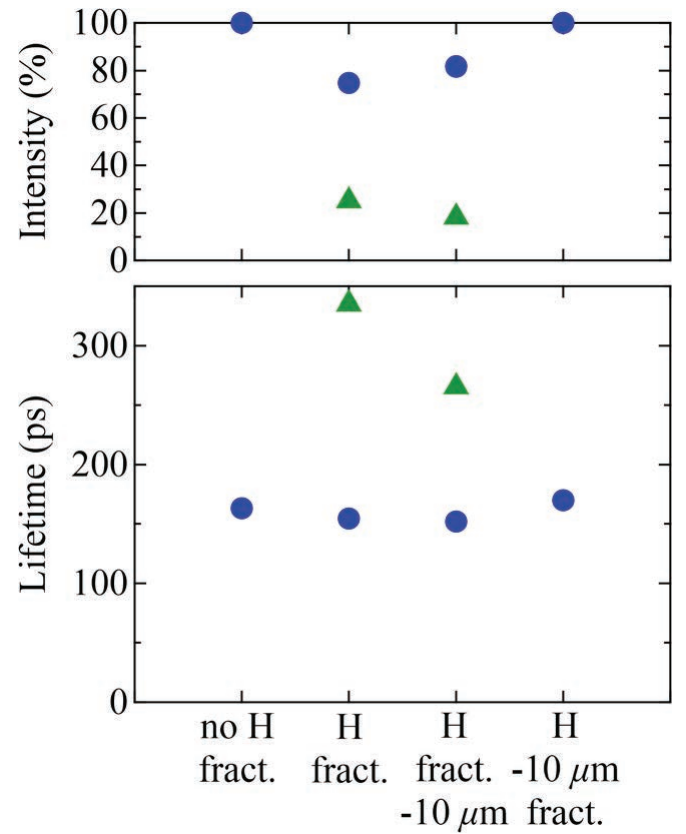

Fig. 5. Positron lifetime and relative intensity from the PALS measurements on the hydrogen-free fractured material, hydrogen-charged fractured sample, hydrogen-charged, fractured and $10-\mu \mathrm{m}$ etched specimen, as well as the hydrogen-charged, $10-\mu \mathrm{m}$ etched and fractured sample. The uncertainties are within the symbol size. Legend: $(\bullet)$ dislocations, ( $\mathbf{\Delta})$ vacancy clusters. (Online version in color.)

hydrogen-enhanced strain-induced vacancies as observed in previous studies. ${ }^{16,19)}$ As a result, a clear difference in the PALS results between the uncharged and charged fractured samples was observed, which is undoubtedly a reflection of the effect of hydrogen. After the hydrogen-charged fractured specimen is $10-\mu \mathrm{m}$ etched, the long lifetime component is still detected but now its value is 265 ps. Therefore, vacancy clusters are found even below the hydrogen-charged layer, although the lifetime indicates that they are smaller in size. In other words, once the charged sample undergoes tensile deformation, the effect of hydrogen appears in the region where hydrogen was not originally present. Such result is a novel finding of the present study obtained by limiting the depth of hydrogen addition and it was not apparent in earlier research ${ }^{16)}$ where, on the other hand, the samples were uniformly charged with hydrogen, e.g. by the highpressure high-temperature $\mathrm{H}_{2}$ gas method. This observation suggests a strain-assisted hydrogen diffusion into the bulk well below $10 \mu \mathrm{m}$, promoted by dislocation dragging and/ or the $\alpha^{\prime}$-phase (where hydrogen diffusion is very fast), that induces the formation of defects in this deeper region. As the XRD spectrum (Fig. 4) shows no martensite phase and somewhat broader $\gamma$-peaks, hydrogen diffusion by dislocation dragging seems more likely. Hence, it is necessary to consider the effect of hydrogen in this deeper region. When the sample is hydrogen-charged, $10-\mu \mathrm{m}$ etched and then fractured, no HE occurs (Fig. 2) and the respective PALS results (Fig. 5) show a single lifetime component of $\sim 160$ ps. This means that just dislocations and no vacancy clusters are formed. This condition resembles that of the hydrogen-free fractured sample and indicates that, because the hydrogencharged layer was etched away before the deformation, hydrogen did not diffuse into the bulk.

A hydrogen-charged sample was pre-strained up to just 
$10 \%$ to study the effect of hydrogen diffusion into the bulk. Figure 6 shows the stress-strain curve of this sample. The XRD measurements of the hydrogen-charged $10 \%$ pre-strained specimen show the formation of the $\alpha^{\prime}$ and $\varepsilon$-phases, which are absent in the hydrogen-free $10 \%$ strained specimen, as seen in Fig. 4. Compared with the hydrogencharged fractured sample, the intensity of the $\varepsilon$-phase hardly changed while that of the $\alpha^{\prime}$-phase increased with increasing strain. This result suggests that the strain-induced martensite transformation is promoted by hydrogen and that the transformation from the $\varepsilon$-phase to the $\alpha^{\prime}$-phase accompanies hydrogen desorption. The PALS results after the $10 \%$ prestrain are shown in Fig. 7. In this case, the PALS spectra are well fitted by three components: bulk, dislocations and hydrogen-enhanced strain-induced vacancy clusters. Note that for this fit the lifetime of the dislocation component was fixed to $160 \mathrm{ps}$ to achieve convergence. Although these results resemble those of the hydrogen-charged fractured specimen, a weak bulk component appears, and the lifetime of the longest component becomes shorter and its intensity decreases, since the sample was subject to just $10 \%$ strain. Note that because the hydrogen distribution and, hence, the defect distribution is not uniform in the sample, the threestate trapping model is not applicable in this case.

After the $10 \%$ pre-strain, the same sample was $10-\mu \mathrm{m}$ etched, and XRD and PALS measurements were carried out again. The XRD measurements show a single-phase pattern characteristic of the $\gamma$-phase and no presence of martensite phases (Fig. 4). The PALS measurements in this case detect just two components: bulk and a new lifetime component of $\sim 180 \mathrm{ps}$, but no vacancy clusters (Fig. 7). Therefore, one may conclude that the $10 \%$ pre-strain was not enough for hydrogen to diffuse to deeper layers and induce the formation of vacancy clusters, as observed in the hydrogen-charged fractured sample. The new lifetime component of $\sim 180$ ps is slightly longer than that of dislocations and cannot be ascribed to monovacancies either, as the latter are unstable at RT. Note that dislocations are expected to form in the hydrogen-charged $10 \%$ pre-strained and $10-\mu \mathrm{m}$ etched specimen, as supported by the slightly broadened $\gamma$-peaks in the XRD spectrum (Fig. 4). Therefore, this $\sim 180$

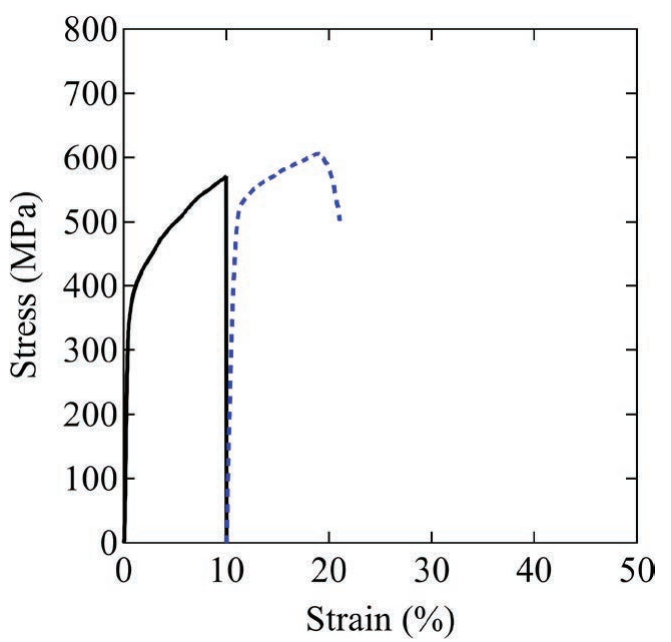

Fig. 6. Stress-strain curves of (-) the hydrogen-charged $10 \%$ pre-strained sample and (- - -) the same sample re-loaded after $10-\mu \mathrm{m}$ etching. (Online version in color.) ps component likely represents the mixed lifetime of dislocations and other unknown defects with a similar lifetime value that cannot be resolved.

After the $10 \%$ pre-strain and $10-\mu \mathrm{m}$ etching, the same sample was strained again until fracture. As shown in Fig. 6 , the ductility significantly declined, which denotes that HE occurred. However, in the SEM image (Fig. 3(c)) the formation of dimples both near the surface and inside the sample and no quasi-cleavage is observed, unlike in the hydrogencharged sample (Fig. 3(b)). The XRD measurements (Fig. 4) show a pattern similar to that of the hydrogen-free fractured sample, with the formation of the strain-induced $\alpha^{\prime}$-phase only. Since the hydrogen-induced $\varepsilon$ and $\alpha^{\prime}$-phases were removed from the surface and yet the sample is hydrogen embrittled, we conclude that these phases are not sufficient conditions for HE to occur and that they cannot be regarded the factors responsible for HE. The PALS spectra of this fractured specimen are well fitted with two components: dislocations and vacancy clusters (Fig. 7). The reappearance of vacancy clusters indicates that they must have developed from the unknown $\sim 180$ ps defects upon further straining. A comparison of the PALS results shows that, although the hydrogen-charged $10-\mu \mathrm{m}$ etched and fracture sample (Fig. 5), and the hydrogen-charged $10 \%$ pre-strained $10-\mu \mathrm{m}$ etched and fractured specimen (Fig. 7) contain the same hydrogen concentration before straining, just the latter is hydrogen-embrittled and exhibits the formation of vacancy clusters. Therefore, the HE of the hydrogen-charged $10 \%$ pre-strained $10-\mu \mathrm{m}$ etched sample proves that hydrogen did diffuse deeper into the bulk and induced the formation of hydrogen-related defects, but dissimilar from vacancy clusters. This unknown $\sim 180$ ps lifetime component must represent the precursors of the defects responsible for HE.

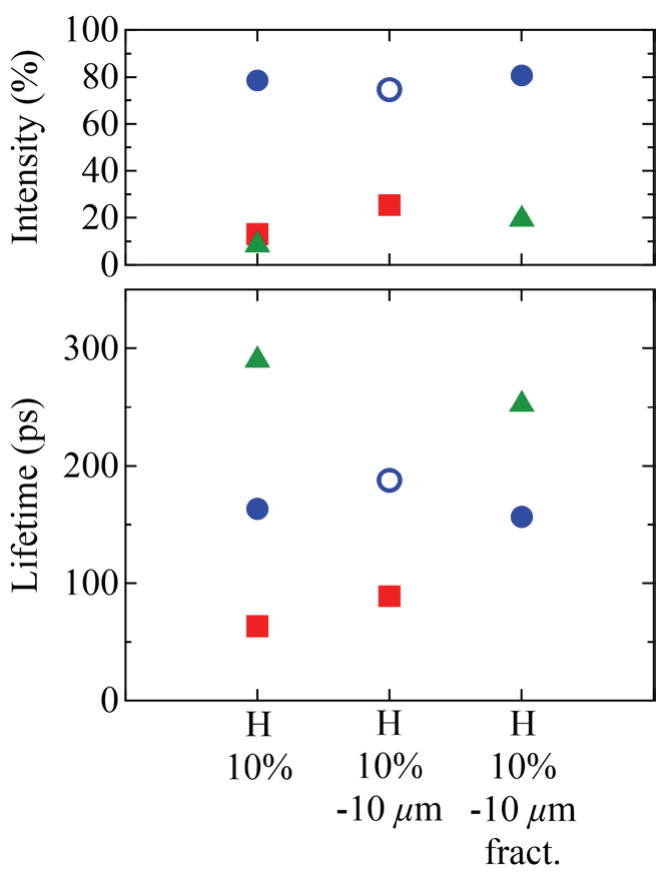

Fig. 7. PALS results of the hydrogen-charged sample after consecutive $10 \%$ pre-strain, $10-\mu \mathrm{m}$ etching and straining until fracture. The uncertainties are within the symbol size.

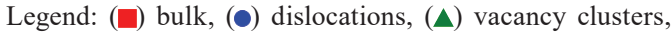
(O) dislocations and vacancy-hydrogen complexes. (Online version in color.) 
To clarify the nature of these unknown defects, we measured the recovery temperature of these defects by PALS measurements during isochronal annealing from RT to $600^{\circ} \mathrm{C}$. Figure 8 shows the results of these PALS measurements for the hydrogen-charged, $10 \%$ pre-strained, $10-\mu \mathrm{m}$ etched sample. It can be seen that the $\sim 180$ ps defects are recovered by annealing at $100^{\circ} \mathrm{C}$ or so. For comparison, the results of the annealing measurements on the hydrogen-
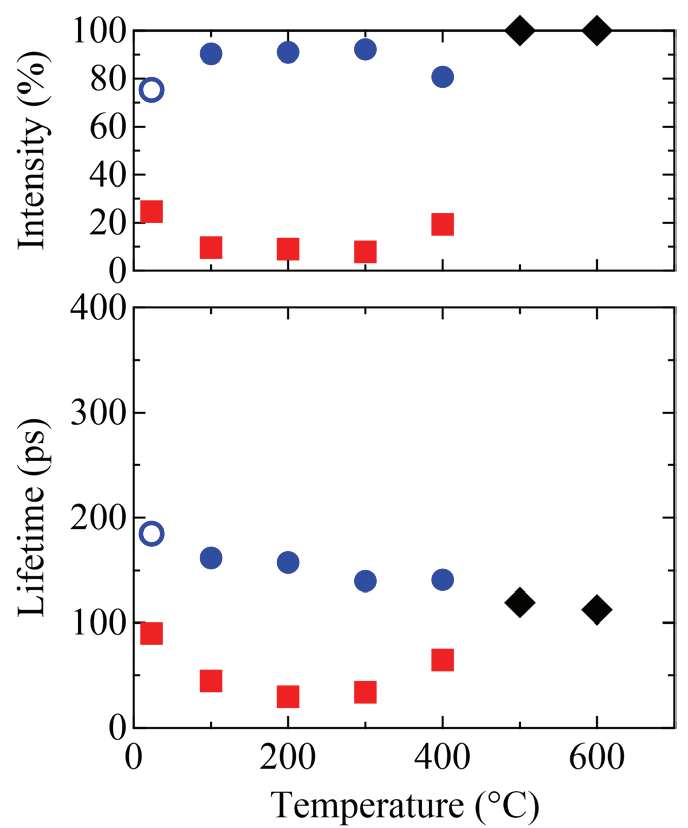

Fig. 8. PALS measurements after annealing of the hydrogencharged, $10 \%$ pre-strained, $10-\mu \mathrm{m}$ etched sample. The uncertainties are within the symbol size. Legend: $(\square)$ bulk, (๑) dislocations, (O) dislocations and vacancy-hydrogen complexes, $(\diamond)$ single component. (Online version in color.)
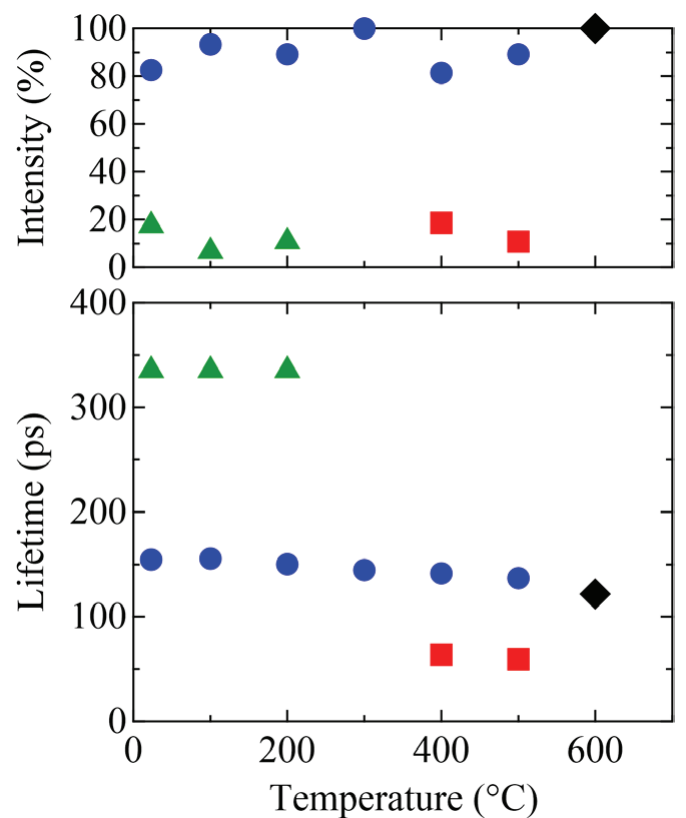

Fig. 9. PALS measurements after annealing of the hydrogencharged fractured sample. The uncertainties are within the

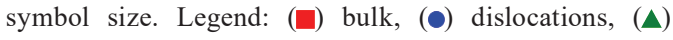
vacancy clusters, $(\bullet)$ single component. (Online version in color.) charged fractured sample are given in Fig. 9. The vacancy cluster and dislocation components are recovered above $200^{\circ} \mathrm{C}$ and $500^{\circ} \mathrm{C}$ or more, respectively, in good agreement with the known recovery temperatures for these defects in $\alpha$-iron. ${ }^{35)}$ From these results, the unknown defects formed in the hydrogen-charged, $10 \%$ pre-strained, $10-\mu \mathrm{m}$ etched sample are attributed to monovacancy-level defects with a higher recovery temperature than that of monovacancies. Since monovacancies are mobile at RT, and yet the $\sim 180$ ps defects are stable at RT, these defects must exist under a different form. Several ab initio calculations have shown that in $\alpha$-iron hydrogen atoms become trapped at monovacancies. ${ }^{6,9,36)}$ Therefore, it is expected that upon hydrogen charge and tensile deformation, hydrogen binds to and stabilizes vacancies. Note that hydrogen coupling to monovacancies is expected to slightly reduce the positron lifetime with respect to that at monovacancies, which is consistent with the measured lifetime of $\sim 180$ ps. By annealing at $100^{\circ} \mathrm{C}$, hydrogen desorbs from the vacancies and monovacancies are recovered. This is consistent with the enhanced hydrogen desorption observed at this temperature from numerous hydrogen-charged steels by thermal desorption analysis. ${ }^{37,38)}$

The present results have shown that by applying stress after electropolishing the hydrogen-charged layer, only dislocations and the strain-induced $\alpha^{\prime}$-martensite phase are formed, and no HE occurs. This proves that hydrogen cannot diffuse to deeper regions than the $10-\mu \mathrm{m}$ charged layer without stress. On the other hand, upon hydrogen charge and application of $10 \%$ strain, dislocations and vacancy clusters are formed. By subsequently removing the hydrogen-charged layer containing a large amount of defects, such as the hydrogen-induced martensite phase and vacancy clusters, the presence of vacancy-hydrogen complexes in the bulk becomes detectable. This highlights the strain-induced diffusion of hydrogen well below the hydrogen-charged layer. Since the vacancy-hydrogen complexes are stable, they are expected to accumulate locally, e.g. in high-strain regions. After application of further strain and the consequent desorption of hydrogen trapped at vacancies, these complexes return to vacancies which diffuse and aggregate into vacancy clusters. As HE is observed in this condition, one may conclude that these vacancy clusters eventually develop into the nuclei of cracks which lead to the brittle fracture. In other words, the vacancy-hydrogen complexes may be regarded as the precursors of the hydrogen-enhanced strain-induced vacancy clusters which trigger the HE.

\section{Conclusions}

In this study, an analysis of the hydrogen-related defects in austenitic stainless steel 304 was carried out by positron annihilation lifetime spectroscopy to determine the defects that control the HE process. We succeeded in measuring and identifying the precursors of the defects responsible for $\mathrm{HE}$ in austenitic stainless steel 304, that had remained undetected so far. This result is of utmost significance for the understanding of the HE mechanism in austenitic stainless steels and represents a first important step in the path for the development of new hydrogen-resistant alloys. Just the electrochemical etching of the hydrogen-charged topmost layer (without any tensile stress) did not result in 
the HE of the sample, whereas etching after application of $10 \%$ strain lead to the HE of the specimen. By removing the hydrogen-charged layer, where a large amount of defects such as the hydrogen-induced martensite phase and vacancy clusters were formed, we successfully detected the hydrogen-induced defects generated in the bulk for the first time. These defects were identified as the precursors of the hydrogen-enhanced vacancy clusters that eventually develop into the brittle fracture. Through isochronal annealing analysis, the nature of those crucial defects was tentatively attributed to the vacancy-hydrogen complexes.

\section{Acknowledgements}

The authors would like to thank M. Hatano, K. Matsumoto and M. Sugeoi in Nippon Steel Stainless Steel Co. for their assistance in the straining and analysis of the samples. This work was supported by the Japan Society for the Promotion of Science (JSPS) KAKENHI Grants no. 18K13980 and no. 19H02742. Financial support under the 27th Steel Research Promotion Grant of the Iron and Steel Institute of Japan was greatly appreciated.

\section{REFERENCES}

1) I. P. Jain: Int. J. Hydrog. Energy, 34 (2009), 7368.

2) E. G. Hertwich, S. Ali, L. Ciacci, T. Fishman, N. Heeren, E. Masanet, F. Nojavan Asghari, E. Olivetti, S. Pauliuk, Q. Tu and P. Wolfram: Environ. Res. Lett., 14 (2019), 043004.

3) M. Nagumo: Fundamentals of Hydrogen Embrittlement: Hydrogen Behavior and Embrittlement Mechanism, Uchida Rokakuho, Tokyo, (2008), 341 (in Japanese).

4) S. Wang, N. Hashimoto and S. Ohnuki: Mater. Sci. Eng. A, 562 (2013), 101.

5) L. Chiari and M. Fujinami: Handbook of Advanced Non-Destructive Evaluation, ed. by N. Ida and N. Meyendorf, Springer International Publishing, Cham, (2019), 1301.

6) Y. Fukai: Materia Jpn., 50 (2011), 465 (in Japanese).

7) K. Sakaki, T. Kawase, M. Hirato, M. Mizuno, H. Araki, Y. Shirai and M. Nagumo: Scr. Mater., 55 (2006), 1031.

8) L. Chiari, A. Nozaki, K. Koizumi and M. Fujinami: Mater. Sci. Eng. A, 800 (2021), 140281

9) Y. Tateyama and T. Ohno: Phys. Rev. B, 67 (2003), 174105.

10) O. Barrera, D. Bombac, Y. Chen, T. D. Daff, E. Galindo-Nava, P. Gong, D. Haley, R. Horton, I. Katzarov, J. R. Kermode, C. Liverani, M. Stopher and F. Sweeney: J. Mater. Sci., 53 (2018), 6251.

11) C. San Marchi, D. K. Balch, K. Nibur and B. P. Somerday: J. Press.
Vessel Technol., 130 (2008), 041401.

12) S. Fukuyama, D. Sun, L. Zhang, M. Wen and K. Yokogawa: J. Jpn. Inst. Met., 67 (2003), 456 (in Japanese).

13) T. Omura, J. Nakamura, H. Hirata, K. Jotoku, M. Ueyama, T. Osuki and M. Terunuma: ISIJ Int., 56 (2016), 405.

14) K. Kamachi, M. Toge and T. Nakanori: J. Soc. Mater. Sci., Jpn., 26 (1977), 322 (in Japanese).

15) N. Narita, C. J. Altstetter and H. K. Birnbaum: Metall. Trans. A, 13 (1982), 1355.

16) M. Hatano, M. Fujinami, K. Arai, H. Fujii and M. Nagumo: Acta Mater., 67 (2014), 342.

17) T. Hajilou, M. S. B. Hope, A. H. Zavieh, N. Kheradmand, R. Johnsen and A. Barnoush: Int. J. Hydrog. Energy, 43 (2018), 12516.

18) J. P. Chateau, D. Delafosse and T. Magnin: Acta Mater., 50 (2002), 1507.

19) K. Takai and M. Kitamura: ASME 2013 Pressure Vessels and Piping Conf. Proc. (American Society of Mechanical Engineers Digital Collection), ASME, New York, (2014), V06BT06A010.

20) M. Nagumo: J. High Press. Inst. Jpn., 48 (2010), 154 (in Japanese).

21) A. Komatsu, M. Fujinami, M. Hatano, K. Matsumoto, M. Sugeoi and L. Chiari: Int. J. Hydrog. Energy, 46 (2021), 6960. https://doi. org/10.1016/j.ijhydene.2020.11.148

22) M. Nagumo and K. Takai: Acta Mater., 165 (2019), 722.

23) M. Mizuno, H. Anzai, T. Aoyama and T. Suzuki: Mater. Trans., JIM, 35 (1994), 703.

24) Y. Mine, Z. Horita and Y. Murakami: Acta Mater., 57 (2009), 2993.

25) M. Enomoto, L. Cheng, H. Mizuno, Y. Watanabe, T. Omura, J. Sakai, K. Yokoyama, H. Suzuki and R. Okuma: Metall. Mater. Trans. E, 1 (2014), 331.

26) T. Omura, K. Kobayashi, M. Miyahara and T. Kudo: Zairyo-toKankyo, 55 (2006), 537 (in Japanese).

27) Y. Sakamoto and H. Katayama: J. Jpn. Inst. Met., 46 (1982), 805 (in Japanese).

$28)$ D. Sun, G. Han, S. Vaodee, S. Fukuyama and K. Yokogawa: Mater. Sci. Technol., 17 (2001), 302.

29) T. Omura, K. Kobayashi, M. Miyahara and T. Kudo: Zairyo-toKankyo, 55 (2006), 139 (in Japanese).

30) S. Kaneko and Y. Sato: J. Surf. Finish. Soc. Jpn., 41 (1990), 203 (in Japanese).

31) J. V. Olsen, P. Kirkegaard, N. J. Pedersen and M. Eldrup: Phys. Status Solidi C, 4 (2007), 4004.

32) M. J. Puska and R. M. Nieminen: J. Phys. F: Met. Phys., 13 (1983), 333.

33) H. Ohkubo, Z. Tang, Y. Nagai, M. Hasegawa, T. Tawara and M. Kiritani: Mater. Sci. Eng. A, 350 (2003), 95.

34) R. N. West: Positrons in Solids, ed. by P. Hautojärvi, SpringerVerlag, Berlin, Heidelberg, (1979), 89.

35) A. Vehanen, P. Hautojärvi, J. Johansson, J. Yli-Kauppila and P. Moser: Phys. Rev. B, 25 (1982), 762.

36) D. A. Mirzaev, A. A. Mirzoev, K. Y. Okishev and A. V. Verkhovykh: Mol. Phys., 112 (2014), 1745.

37) K. Takai: Zairyo-to-Kankyo, 60 (2011), 230 (in Japanese).

38) S. Yamasaki, D. Hirakami and T. Manabe: Shinnittetsu Sumikin Giho, 406 (2016), 37 (in Japanese) 